\title{
Oriented Asymmetric Kernels for Corner Detection
}

\author{
Hasan Abdulrahman, Baptiste Magnier and Philippe Montesinos, \\ Ecole des Mines d'Alès, Parc scientifique Georges Besse, 30000 Nîmes, France \\ E-mail: hasan.abdulrahman@mines-ales.fr, baptiste.magnier@mines-ales.fr, philippe.montesinos@mines-ales.fr
}

\begin{abstract}
Corners and junctions play an important role in many image analysis applications. Nevertheless, these features extracted by the majority of the proposed algorithms in the literature do not correspond to the exact position of the corners. In this paper, an approach for corner detection based on the combination of different asymmetric kernels is proposed. Informations captured by the directional kernels enable to describe precisely all the grayscale variations and the directions of the crossing edges around the considered pixel. Compared to other corner detection algorithms on synthetic and real images, the proposed approach remains more stable and robust to noise than the comparative methods.
\end{abstract}

Index Terms-Oriented filters, detection of corners.

\section{INTRODUCTION AND RELATED WORK}

In digital images, corners and junctions constitute important landmarks because they get affluent information like delimitation of objects. A pixel is considered a corner or a junction when two or more edges meet each other and refer to the point where several different contour orientations exist. These orientations could be estimated using structure tensors, voting tensors or oriented kernels. Thereafter, a non exhaustive review of corner detection methods is presented. Then, oriented kernels are described. Finally, a new corner detection method based only on oriented kernels is described.

\section{A. Corner detection techniques}

In the literature, several approaches have been developed to detect corners and junctions: (i) involving contour chains, (ii) using templates or, (iii) by image filtering techniques.

Traditional contour based corners methods focus on the processing of binary edges, by searching points having curvature in contour chains or in line segment intersections [21] [2]. This type of approach might be insufficient for applications in natural images because the accuracy of corner localization relies on edge detection performance (filtering, threshold(s)).

Another way to extract these feature points involves gradient orientation histogram [24] or templates in which all pixel values are compared with that of center pixel(s) of the shape template. In the wellknow SUSAN (Smallest Univalue Segment Assimilating Nucleus [23]), a corner is extracted in comparing every pixel inside a circular mask with the central pixel. On the contrary, for the FAST (Features from Accelerated Segment Test [19]) method, a point is considered a corner only if there are several pixels in the path of a circular template which are higher or lower than the value determined by the central pixel. These corner detection methods do not require any spatial derivatives; however, they do not lead directly to the position of the corner (i.e. the pixel having several local orientations as in Fig. 1 (d) and (e)).

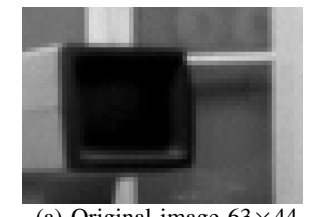

(a) Original image $63 \times 44$

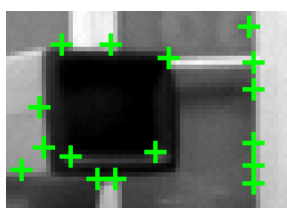

(d) SUSAN [23]

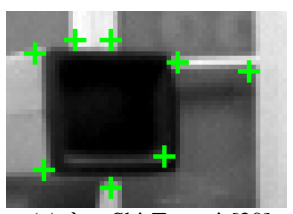

(g) $\lambda_{2}$, Shi-Tomasi [20]

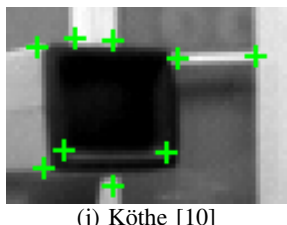

(j) Köthe [10]

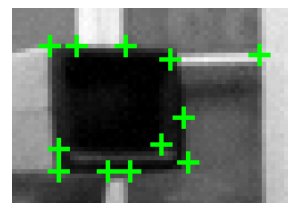

(b) Contour-based [24]

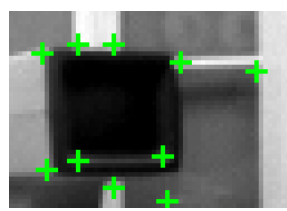

(e) FAST [19]
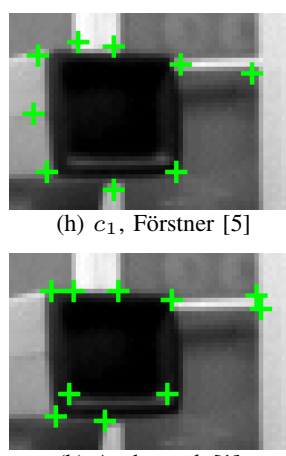

(k) Aach et al. [1]

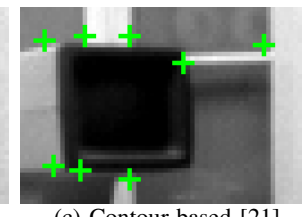

(c) Contour-based [21]

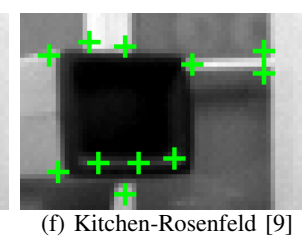

(f) Kitchen-Rosenfeld [9]
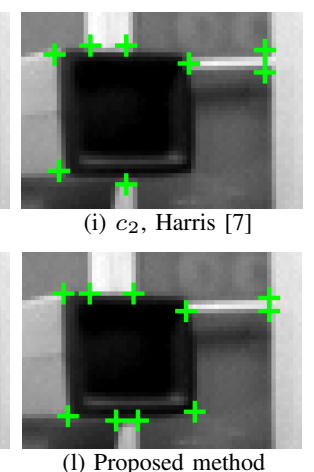

(l) Proposed method

Fig. 1. Corner detected involving several methods. The standard deviation used for the image derivatives and for the structure tensor $J_{\rho}$ are: $\sigma=\rho=1$. For the developed method: $\sigma_{\eta}=1$ and $\sigma_{\xi}=3, L=3$ and $P=5$.

As far as, image filtering is oncerned, the feature detectors operate directly on image intensities. Corners are defined by the combination of the gradient magnitude and points having maximum curvature of the image surface. A pioneer work in this domain remains Kitchen-Rosenfeld algorithm which involves first and second order image derivatives in the cornerness computation [9]. Using of second derivatives introduces false alarms in the results of this method because second image derivatives are better adapted for ridge detection. Indeed, the computation of only the first image derivatives informs on the local structure at a pixel by examining also the data in the neighborhood. Moreover, integrating the gradient information in the neighborhood of the pixel (i.e. the correlation) brings indications about whether the pixel must be considered an edge or a corner. This selection is given by involving a $2 \times 2$ symmetrical structure tensor. The derivation of a scalar image $I$ is called the image gradient and is noted by $\nabla I=\left(I_{x}, I_{y}\right)^{t}$ in which $I_{x}$ and $I_{y}$ represent the image derivatives in $x$ and $y$ directions respectively (usually calculated by means of Gaussian derivative filters with a standard deviation of $\sigma \in \mathbb{R}_{+}$, see Fig. 2(a)). Involving a smoothing Gaussian kernel 
$G_{\rho}$ of standard deviation $\rho$, the first-order structure tensor $J_{\rho}$ is given by $J_{\rho}(\nabla I)=G_{\rho} * \nabla I \cdot \nabla I^{t}$. The scale of the neighborhood information is given by $G_{\rho}$. When $J_{\rho}$ possesses two positive eigenvalues, then the pixel is considered having at least two distinguished orientations, therefore a corner or a junction. This isotropic cornerness measurement is given by the second eigenvalue denoted $\lambda_{2}$ [20][3] or could be estimated by $c_{1}=\frac{\operatorname{det}\left(J_{\rho}\right)}{\operatorname{tr}\left(J_{\rho}\right)+k}$ [5] or $c_{2}=\operatorname{det}\left(J_{\rho}\right)-k \cdot \operatorname{tr}\left(J_{\rho}\right)$ [7], with $k>0$ (see also the diffusion scheme in [18] which preserves corners using $\lambda_{2}$ ). Feature detection using the linear structure tensor $J_{\rho}$ is insufficient in the presence of more than one dominant direction. Depending on its smoothing parameter $\rho$, this tensor representation is robust under noise, but generally the localization of the detected corner lacks precision. Indeed, according to the scale $\rho$ and the image derivatives estimated by convolution with Gaussian kernels, the detected location of a corner tends to shift as $\rho$ increases [4], as illustrated Figs. 1(g), (h) and (i). To bypass this weakness, several solutions have been proposed in the literature as nonlinear structure tensors [3] or tensor voting [15]. In [10], the cornerness measurement $\lambda_{2}$ is propagated using hourglass-shaped filter instead of the Gaussian mask for data-adaptive smoothing at the crossing edge position. Finally, in [1], double local orientations for corners and junctions are extracted by: (1) involving $J_{\rho}$ to detect regions containing double orientations and (2) the computation and combinations of the eigenvalues of a $3 \times 3$ tensor. This method is more precise than previous mentioned approaches, as shown in Fig. 1(k).

In this work, corners and junctions are directly extracted involving only a combination of asymmetric oriented kernels.

\section{B. Oriented Kernels and Edges Directions}

Oriented filters were designed to capture multidirectional gray intensity variations [6] [8]. Indeed, they consist in finding the orientation where the derivative corresponds to the maximum response. The concept was generalized in [17] by decomposing a given filter kernel optimally in a set of basis filters which approximates an Anisotropic Gaussian Kernel (AGK):

$$
A G K_{\sigma_{\xi}, \sigma_{\eta}}(x, y)=\frac{1}{2 \pi \cdot \sigma_{\xi} \cdot \sigma_{\eta}} \cdot e^{-\frac{1}{2}\left(\frac{x^{2}}{\sigma_{\xi}^{2}}+\frac{y^{2}}{\sigma_{\eta}^{2}}\right)},
$$

where $(x, y) \in \mathbb{R}^{2}$ represent the pixel coordinates, $\sigma_{\eta}$ and $\sigma_{\xi}$ are referred to the Gaussian scale and to the anisotropic factor respectively (Fig. 2(b)). The AGK can be oriented [21] but, possesses a common shortcoming, as a matter of fact, only one $\pi$-periodic orientation is extracted efficiently [17], so the impossibility of these filters to estimate in a relevant way several coexisting orientations at the same pixel (see Fig. 1 (c)).

Contrary to the templates remembered above, wedge [22][16] or asymmetric oriented filters [11][14] sound better suited for a purpose like multiple edge directions detection or modeling a template. Thus, corner analysis requires finding maxima in filter responses when these $2 \pi$-periodic filters are successively steered in different directions. The equation of

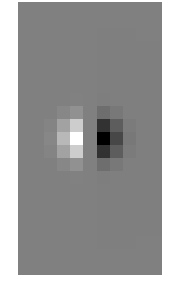

(a) Isotropic, $\sigma=1$

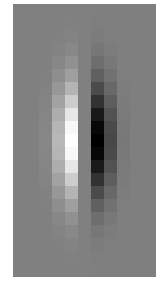

(b) AGK

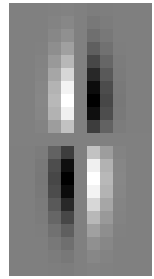

(c) Endstop kernel

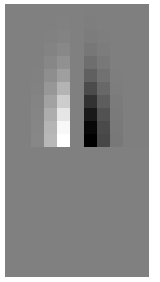

(d) HGK

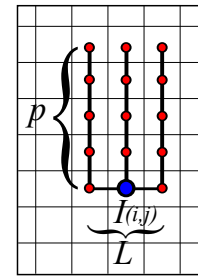

(e) IRON, $L=3$ and $P=5$
Fig. 2. Different discretized 2D derivative Gaussian kernels and representation of the IRON filter. (b), (c) and (d) with $\sigma_{\eta}=1$ and $\sigma_{\xi}=3$. The Anisotropic endstop filter is equivalent to the derivative of the AGK along the Y direction.

the anisotropic edge detector based on Half Gaussian Kernels (HGK) derivative is given by:

$$
\operatorname{HGK}_{\sigma_{\xi}, \sigma_{\eta}}(x, y)=-H(y) \cdot \frac{x}{\sigma_{\eta}} \cdot G_{\sigma_{\xi}, \sigma_{\eta}}(x, y),
$$

where $H$ corresponds to the Heaviside function, illustrated in Fig. 2(d). By rotating the image [11], maxima of the filter responses indicate the directions of the edges from 0 to $2 \pi$ [11][12]. In addition, HGK and wedge steerable filters responses easily caracterize corners and junctions. Nevertheless, inside homogeneous/noisy regions, due to the isophotes, i.e., curves of the image surface of constant intensity, these directions become unpredictable. Perona shows in [17] that the combination of the endstop kernel (see Fig.2 (c)) with the HGK enables a junction and corner characterization. Indeed, the response of the combination is null along a straight contour whereas the response has maxima along the directions of the contours forming a junction or a corner (illustrated in Fig. 3). In order to avoid false corners or junctions, another solution proposed in [12] is to align the directions of the HGK when the gradient value is weak. However, near edges, the gradient generated by the HGK remains not so weak and disturbs the corner detection by creating a halo of acute angles around the contours (note that this remains the same problem using the endstop kernel). Fig. 3 (bottom) and Fig. 4 (d) illustrate this phenomenon, corners are correctly localized, however, close to the edge, HGK creates an angle which can be considered a corner.

\section{A NEW METHOD OF CORNER EXTRACTION}

The main idea of this new approach is to combine the HGK with an asymmetric filter computing the homogeneity along edges. On the one hand, the maxima responses of the HGK indicate the directions ( $2 \pi$ periodic) of the edges. On the other hand, the oriented variance determines if the directions of the maxima of the HGK corresponds to edges or other types of pixels (texture, homogeneous region etc.).

\section{A. Oriented Filter of Grayscale Homogeneity}

The asymmetric IRON (Isotropic and Recursive Oriented Network) filter estimates the homogeneity in multiple local directions [14]. This filter consists in a network of several parallel lines in which a homogeneity is computed and enables 
an estimation of edge directions modulo $2 \pi$. The variance for a pixel located at $(x, y)$ on the network is computed by:

$$
\operatorname{IRON}(x, y)=\frac{1}{L} \sum_{j=0}^{L}\left(\frac{1}{P} \sum_{i=0}^{P}\left(I(i, j)^{2}\right)-\left(\frac{1}{P} \sum_{i=0}^{P} I(i, j)\right)^{2}\right)
$$

Here, $L$ represents the number of lines where the variance is computed and $P$ the number of points per line. Fig. 2(e) represents an example of an asymmetric IRON filter. Computationally, the rotation of the image is applied at some discretized orientations from 0 to $2 \pi$ before applying the IRON filters. Some examples of IRON filter signals are available in Fig. 3 fifth column, values of the IRON are close to 0 in the edges directions and it is shown in [14] that the detections of edges directions stay precise in the presence of noise.

\section{B. A Combination of Homogeneity and Edge Strength}

The HGK and the asymmetric IRON are 1-side kernels, so they are steered in $2 \pi$ directions. Moreover, the response of the HGK corresponds to maxima along the directions of the edges while the response of the IRON has minima along these same directions. Hence, the combination at the orientation $\theta \in$ $[0 ; 2 \pi$ [ between the HGK and IRON is straightforward:

$$
\mathcal{S}(x, y, \theta)=\frac{\operatorname{HGK}(x, y, \theta)}{\epsilon+\operatorname{IRON}(x, y, \theta)}, \quad \text { with } \epsilon \in \mathbb{R}_{*}^{+},
$$

where $\operatorname{HGK}(x, y, \theta)$ and $\operatorname{IRON}(x, y, \theta)$ represent, respectively the HGK and IRON responses in a rotated image of angle $\theta$. Finally, $\epsilon$ corresponds to a constant avoiding a division by 0 . The corner detection is equivalent to analyze the resulting signal $\mathcal{S}$ for each pixel. The polar curves in Fig. 3 indicate the modulus of the different kernels responses. Moreover, the extrema of $\mathcal{S}$ indicate the precise directions of the edges for contour and corner points. As illustrated in Fig. 4 (e), at a distance between 1 and $2 \sigma_{\eta}$ pixels, the extrema of $\mathcal{S}$ correspond to directions which are parallel to the edge directions. Finally, in order to compute the variance and the oriented derivative on the same neighborhood, the spacial influence of the IRON filter is inserted in the support of the HGK, i.e. $3 P \leqslant \sigma_{\xi}$ and $3 L \leqslant \sigma_{\eta}$.

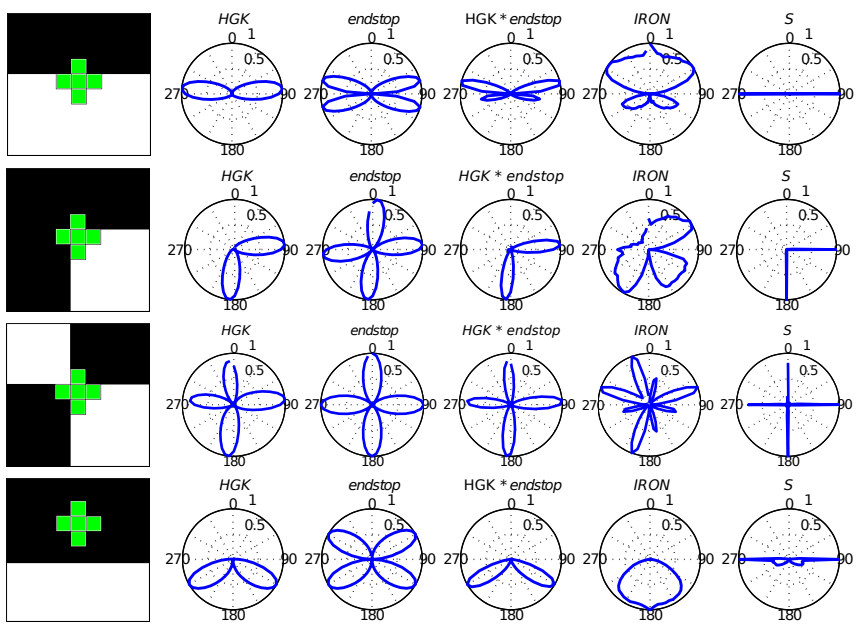

Fig. 3. Modulus of the energy of the different oriented kernels and their combinations (in degrees and normalized signals).

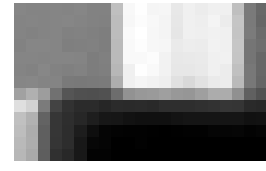

(a) Original image $13 \times 21$

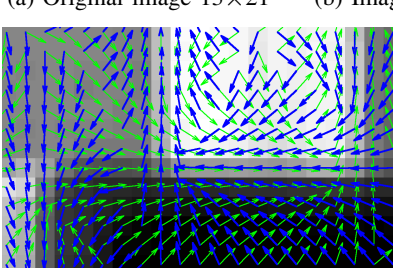

(d) Directions of the extrema of HGK (b) Image of $\mathcal{C}$ (Eq. 5)

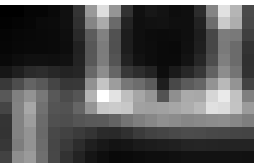

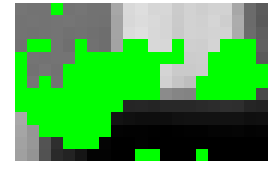

(c) Image of $\frac{\pi}{3} \leqslant \beta \leqslant \frac{2 \pi}{3}$
Fig. 4. Direction field. (a) Cropped image of Fig. 1(a).

\section{Angle Selection and Corner Extraction}

The IRON energy is always positive while the HGK filter corresponds to an oriented derivative, so its responses are either positive, or negative. Consequently, the signal $\mathcal{S}$ possesses positive/negative values when $\mathrm{HGK}$ is positive/negative (see Fig. 5). To obtain the cornerness measure $\mathcal{C}$ as in Fig. 4 (b), the global extrema of $\mathcal{S}$ are combined:

$$
\left\{\begin{aligned}
\mathcal{C}(x, y) & =\max _{\theta \in[0,2 \pi[} \mathcal{S}(x, y, \theta)-\min _{\theta \in[0,2 \pi[} \mathcal{S}(x, y, \theta) \\
\theta_{1}(x, y) & =\underset{\theta \in[0,2 \pi[}{\arg \max }(\mathcal{S}(x, y, \theta)) \\
\theta_{2}(x, y) & =\underset{\theta \in[0,2 \pi[}{\arg \min }(\mathcal{S}(x, y, \theta)) \\
\beta(x, y) & = \begin{cases}\left|\theta_{1}(x, y)-\theta_{2}(x, y)\right|, \text { if }\left|\theta_{1}(x, y)-\theta_{2}(x, y)\right| \leqslant \pi \\
2 \pi-\left|\theta_{1}(x, y)-\theta_{2}(x, y)\right| & \text { elsewhere. }\end{cases}
\end{aligned}\right.
$$

Once $\mathcal{C}, \theta_{1}$ and $\theta_{2}$ have been obtained, the corners can be easily extracted in two steps: (i) by selecting pixels where $\beta$, the angle formed by $\theta_{1}$ and $\theta_{2}$, corresponds to a desired angular sector followed by (ii) thresholding the local maxima of $\mathcal{C}$. Finally, for a pixel belonging to a straight contour, the value of $\mathcal{C}$ is high (see Fig. 4 (b)) while $\beta$ corresponds to an open angle $\approx \pi$ ((illustrated in Fig. 5 and $4(\mathrm{c})$ ); so it is not considered a corner point.

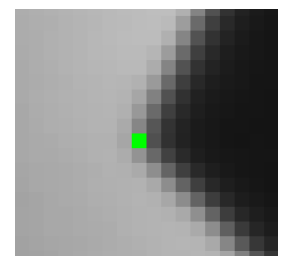

Corner point

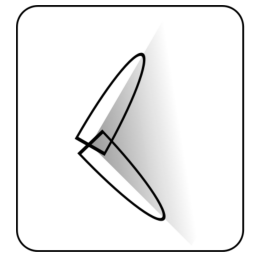

Directions for HGK maxima

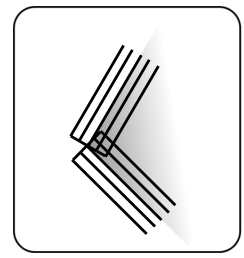

Directions for IRON minima
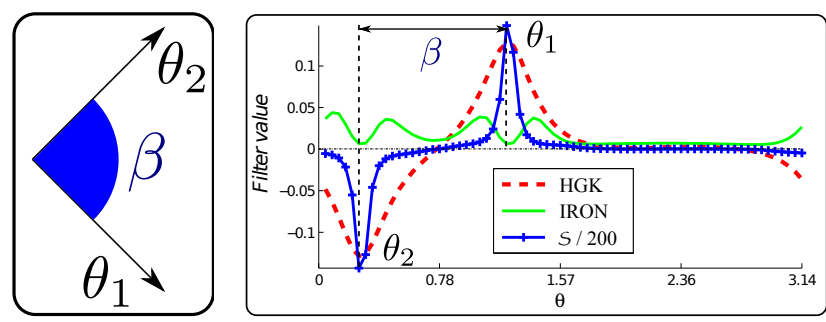

Fig. 5. The minimum and maximum of the signal $\mathcal{S}$ corresponds to the two directions of the edges and $\beta$ to the angular sector between the $\theta_{1}$ and $\theta_{2}$ directions. 

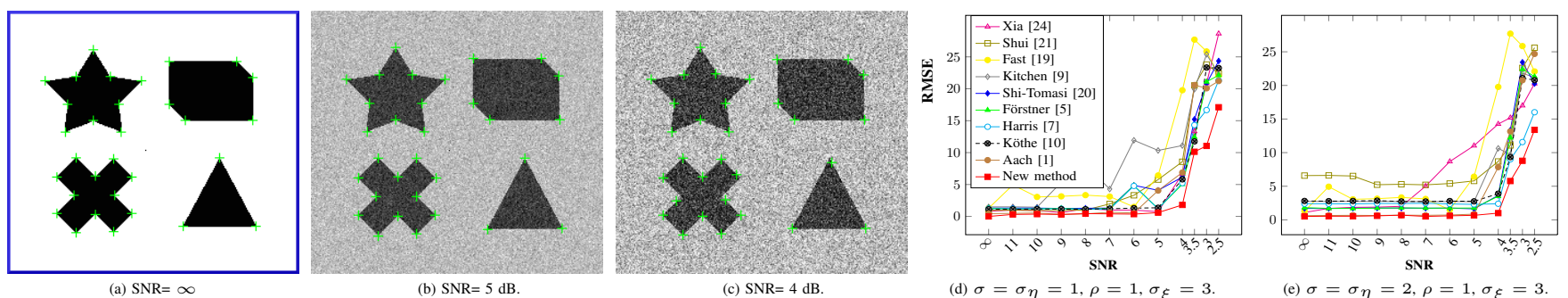

Fig. 6. RMSE in function of the noise level. (a)-(c) contains the corner detected by the new algorithm with $L=3$ and $p=5$.

\section{EXPERIMENTS AND EVALUATION}

The experiments are carried out on synthetic images and real images to compare corner detectors. All the images and other results are available on the website: http://hkaljaf.wixsite.com/ hasanabdulrahman/corners-and-junction-detection.

First, the 31 "best" corners are extracted from the synthetic image by corner detectors. These corners are composed of acute and obtuse angles. Then, the Root-Mean-Square Error (RMSE) is computed between the 31 true corners and the extracted features. Considering $T_{c}$ and $D_{c}$ the set of true and detected corners respectively:

$$
\mathrm{RMSE}=\sqrt{\frac{1}{\operatorname{card}\left(T_{c}\right)+\operatorname{card}\left(D_{c}\right)} \cdot\left(\sum_{p \in D_{c}} d_{T_{c}}^{2}(p)+\sum_{p \in T_{c}} d_{D_{c}}^{2}(p)\right) .}
$$

For a pixel $p \in D_{c}, d_{T_{c}}(p)$ represents the minimal Euclidean distance between $p$ and $T_{c}$, whereas if $p \in T_{c}, d_{D_{c}}(p)$ corresponds to the minimal distance between $p$ and $D_{c}$. Note that the two distances $d_{T_{c}}$ and $d_{D_{c}}$ are recorded [13]. Indeed, only the calculation of D can favour an algorithm where the detected corners are agglutinated around a single true point. Thus, ten corner detection approaches are compared in terms of the noise level, which is indicated by the Signal-to-Noise Ratio (SNR), as illustrated in Figs. 6 (a), (b), (c) and (d).

Curves in Figs. 6(d-e) show the RMSE in function of the SNR when the standard deviation for the Gaussian derivative is the same for all methods excepted FAST ( $\sigma_{\eta}=1$ in (d) and $\sigma_{\eta}=2$ in (e) in our case). Indeed, the standard deviation is the same for all detectors in order to compare them together, even for gradient orientation histogram technique [24] and contourbased method [21]. The proposed method achieves the best results in term of RMSE for all the noise levels, red curves in Figs. 6(d-e). The shape of the considered filters enables to locate the corners at the correct position even though the noise is strong (see Fig. 6(d) with SNR=4dB). Note that results for [10] are in sub-pixels resolution and merged to the original size for the evaluation process without lose detected points.

The same evaluation is led by changing the standard deviation for the Gaussian derivative $\left(\sigma=\sigma_{\eta}=2\right)$ for all tested methods. The curve in Fig. 6 (f) illustrates the error measures. As pointed out in Section I-A, the scale of isotropic detectors affects the localization of detected corners. Note that the contour based on anisotropic Gaussian kernels is not robust to the detection of acute corners because these kernels delocalize strongly the corner points in the edge detection stage (see [11]). On the contrary, the proposed method remains stable (less than 1 pixel RMSE measure compared to $\sigma_{\eta}=1$ in the previous case). As far as acute angles are concerned, the half kernels are able to select the two directions of the edges and then qualify these pixels as corners. Finally, the performance of the new method is due to the HGK combined with the IRON filter which corresponds to thin filters, allowing a precise direction of contours, and thus of corners.

The first real image in Fig. 1(a) is composed of thin structures with a blur. Despite that, Fig. 1(1) illustrates very clearly that the new corner detection method has better accuracy than the ten other corner detectors.

The last experiment presented in this study focuses on the 'lab' image in Fig. 7(a). This image contains several corners of different type of angles and blurred edges. To make the comparison easier, the 280 best interest points are extracted for each algorithm. On the one hand, the orientation histogram technique [24], the contour-based method [2] and the tensorial approaches [20][5][7][10][1] fail to detect the majority of corners of obtuse angles (e.g. carpets on the floor) while they detect a lot of features concerning small objects as in the top right-hand of the image. On the other hand, Fig. 7 (f) shows that the performance of the proposed method is more efficient to detect the features. In order to capture acute and obtuse corner, the angular sector belongs to $\left[\frac{\pi}{6}, \frac{5 \pi}{6}\right]$. Such an angular sector is enough to detect only desired features only if the spacial support of the IRON filter is include in the spacial (Fig. 5 top right) support of the half Gaussian kernel (Fig. 5 top middle). These results using the combination of oriented half kernels and IRON filter illustrate reliable and promising results, even when dealing with blurred images.

\section{REFERENCES}

[1] T. Aach, C. Mota, I. Stuke, M. Mühlich, E. Barth, Analysis of superimposed oriented patterns, IEEE TIP, Vol. 15(12), pp. 3690-3700, 2006.

[2] M. Awrangjeb, G., Lu, C.S. Fraser, Performance comparisons of contourbased corner detectors, IEEE TIP, Vol. 21(9), 4167-4179, 2012.

[3] T. Brox, J. Weickert, B. Burgeth, and P. Mrázek, Nonlinear structure tensors, IVC, Vol. 24(1), pp. 41-55, Elsevier, 2006.

[4] R. Deriche and G. Giraudon, A computational approach for corner and vertex detection, IJCV, Vol. 10(2), pp. 101-124, 1993.

[5] W. Förstner, A feature based correspondence algorithm for image matching, Int. Arch. of Photogrammetry and Remote Sensing, Vol. 26(3), pp. $150-166,1986$.

[6] W. T. Freeman and E. H. Adelson, The design and use of steerable filters, IEEE TPAMI, Vol. 13(9), pp. 891-906, 1991.

[7] C. Harris and M. Stephens, A combined corner and edge detector, Alvey Vis. Conf., Citeseer, Vol. 15, p. 50, 1988.

[8] M. Jacob, M. Unser, Design of steerable filters for feature detection using Canny-like criteria, IEEE TPAMI, Vol. 26(8), pp. 1007-1019, 2004.

[9] L. Kitchen and A. Rosenfeld, Gray-level corner detection, Tech. Rep., DTIC Document, 1980.

[10] U. Köthe, Edge and junction detection with an improved structure tensor, Joint Patt. Rec. Symp., Springer, pp. 25-32, 2003.

[11] B. Magnier, P. Montesinos, and D. Diep, Fast anisotropic edge detection using gamma correction in color images, IEEE ISPA, pp. 212-217, 2011

[12] B. Magnier and P. Montesinos, Oriented half gaussian kernels and anisotropic diffusion, VISAPP, Vol. 1, pp. 73-81, 2014. 


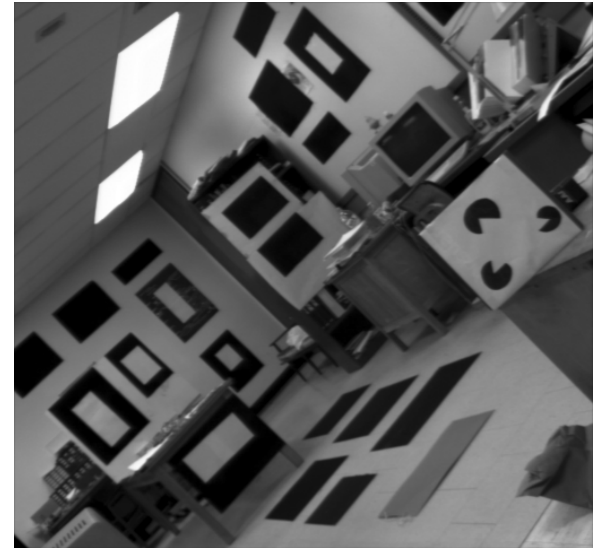

(a) Original image $512 \times 484$

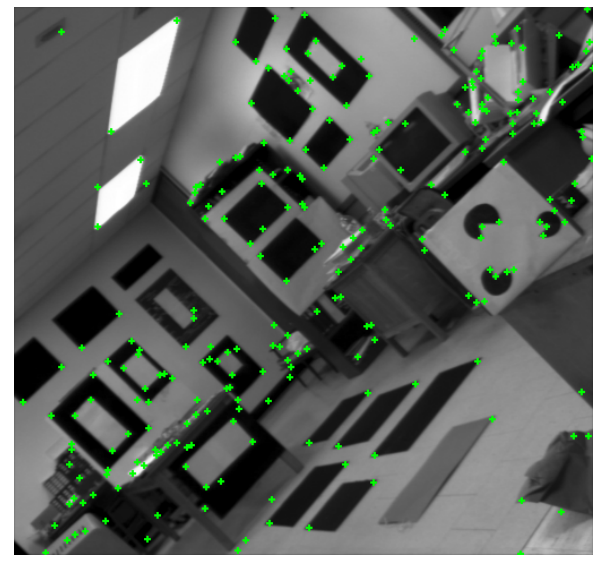

(d) $\lambda_{2}$, Shi-Tomasi [20]

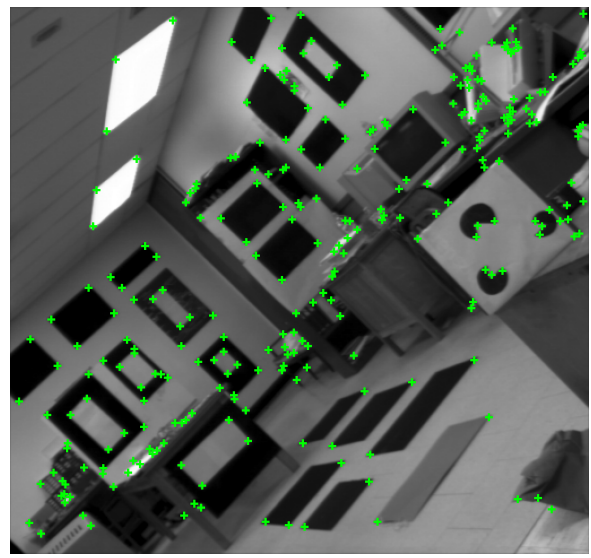

(g) Koethe [10]

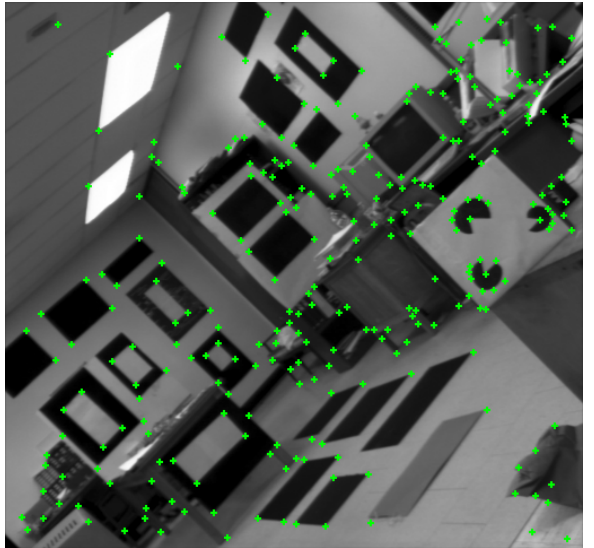

(b) Contour-based [24]

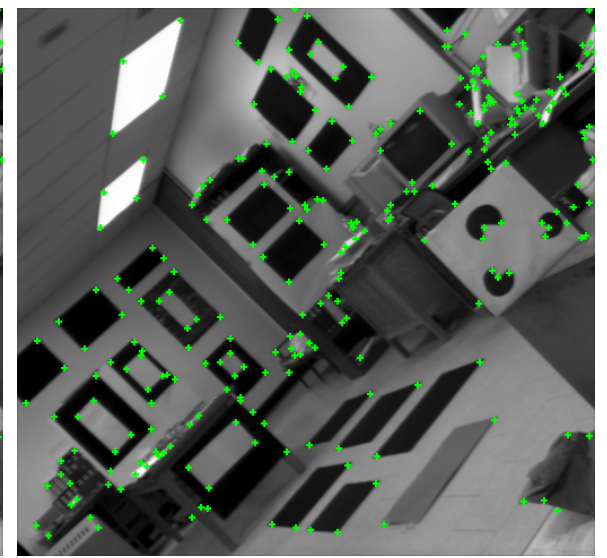

(e) $c_{1}$, Förstner [5]

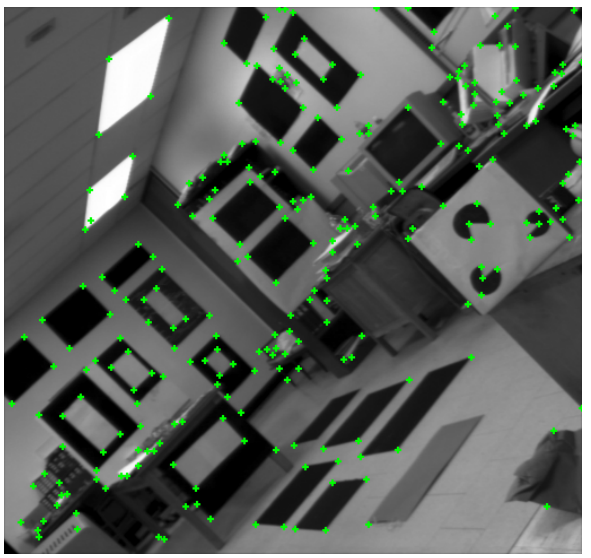

(h) Aach et al. [1]

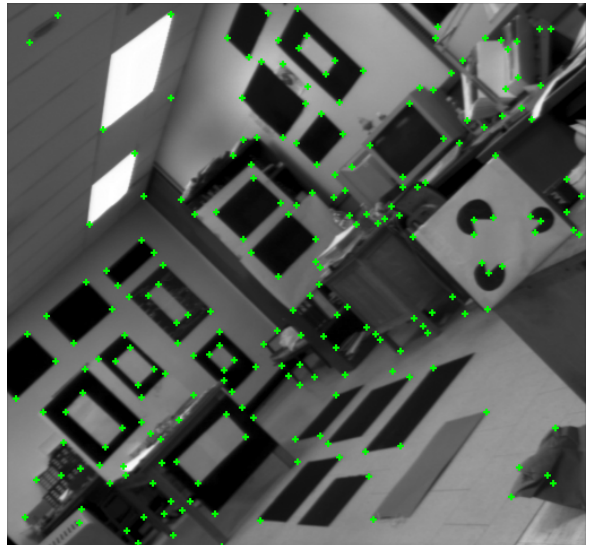

(c) CPDA [2]

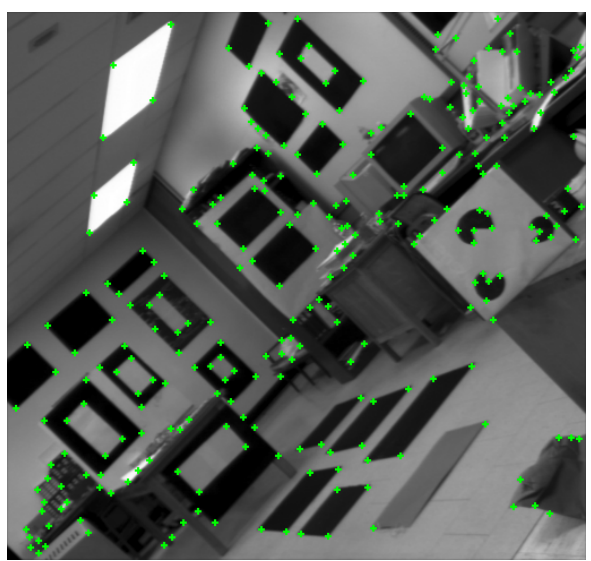

(f) $c_{2}$, Harris [7]

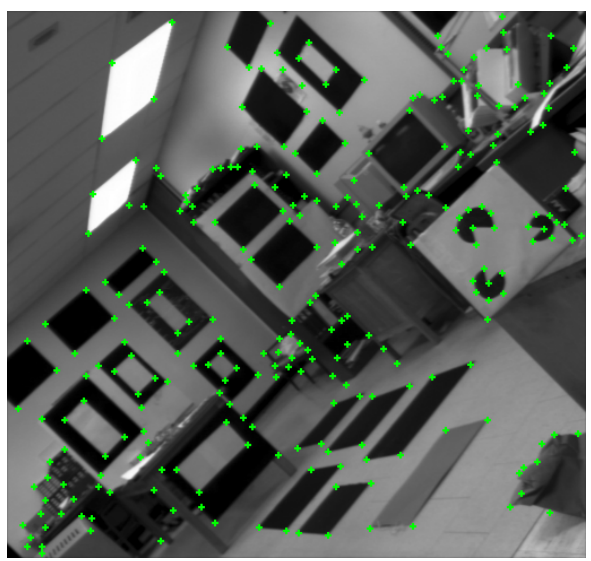

(i) Proposed method

Fig. 7. Corner detection involving several methods: 280 best points for each method. The standard deviation used for the image derivatives and for the structure tensor $J_{\rho}$ are: $\sigma=1.5$ and $\rho=1$. For the developed method: $\sigma_{\eta}=1.5$ and $\sigma_{\xi}=5, L=3$ and $P=5$.

[13] B. Magnier, Edge Detection: A Review of Dissimilarity Evaluations and a Proposed Normalized Measure, Mult. Tools and Applications, 2017.

[14] F. Michelet, J.-P. Da Costa, O. Lavialle, Y. Berthoumieu, P. Baylou, and C. Germain, Estimating local multiple orientations, Signal Proc., Vol. 87(7), pp. 1655-1669, 2007.

[15] R. Moreno, L. Pizarro, B. Burgeth, J. Weickert, M. A. Garcia, D. Puig, Adaptation of tensor voting to image structure estimation, New Dev. in the Visualization and Proc. of Tensor Fields, pp. 29-50. Springer, 2012.

[16] M. Mühlich, T. Dahmen, and T. Aach, Doubly-rotated matched filtering, IEEE EUSIPCO, pp. 2169-2173, 2007.

[17] P. Perona, Steerable-scalable kernels for edge detection and junction analysis, IVC, Vol. 10(10), pp. 663-672, 1992.

[18] K. O. Riedel, Corner-preserving anisotropic diffusion and junction detection using the structure tensor, . Mustererkennung, pp. 164-171, Springer Berlin Heidelberg, 1999.
[19] E. Rosten, R. Porter, and T. Drummond, Faster and better: A machine learning approach to corner detection, IEEE TPAMI, Vol. 32(1), pp. 105-119, 2010.

[20] J. Shi and C. Tomasi, Good features to track, IEEE CVPR, pp. 593600, 1994.

[21] P.-L. Shui and W.-C. Zhang, Corner detection and classification using anisotropic directional derivative representations, IEEE TIP, Vol. 22(8), pp. 3204-3218, 2013.

[22] E. P. Simoncelli and H. Farid, Steerable wedge filters for local orientation analysis, IEEE TIP, Vol. 5(9), pp. 1377-1382, Citeseer, 1996.

[23] S. M. Smith and J. M. Brady, SUSAN a new approach to low level image processing, IJCV, Vol. 23(1), pp. 4578, Springer, 1997.

[24] G.S. Xia, J. Delon, and Y. Gousseau, Accurate junction detection and characterization in natural images, IJCV, Vol. 106(1), pp. 3156 Springer, 2014 